\title{
Schulsozialarbeit braucht mehr Rechte
}

\author{
Ein Berufsfeld der Sozialen Arbeit zwischen Lerntradition und Lebenswelt
}

Susanne Hartmann-Hanff

Trotz jabrzehntelanger Annäherungsversuche der Jugendhilfe an die Schule ist es bisher nicht gelungen, Soziale Arbeit in dieser Bildungseinrichtung flächendeckend als Regelangebot zu verankern. Dabei könnte Schulsozialarbeit zur Optimierung der schulischen Sozialisation beitragen.

Das Arbeitsfeld Schulsozialarbeit ist bislang formaljuristisch unzureichend geregelt, begrifflich unübersichtlich und organisatorisch uneinheitlich. Gäbe es im Sinne der Kinderrechtskonvention der Europäischen Union eine ernstzunehmende politische Absicht, die gesellschaftliche und berufliche Integration von Kindern und Jugendlichen zu fördern, müsste es eine sanktionsbewehrte, verpflichtende Abstimmung des SGB VIII mit den korrespondierenden Schulgesetzen der Länder geben, und zwar im Sinne eines verbindlichen gesetzlichen Auftrages zur Schulsoziarbeit für Jugendhilfe und Schule.

Die Ausdifferenzierung der Begrifflichkeit »Schulsozialarbeit « als » Jugendhilfe und Schule «, "schulbezogene Jugendsozialarbeit ", "schulbezogene Jugendhilfe « oder auch "Sozialarbeit in Schulen « irritiert meines Erachtens nach unnötig die fachliche Debatte. Selbst wenn damit verschiedene paradigmatische und organisatorische Schwerpunktorientierungen verbunden sind, sehe ich wegen des Bekanntheitsgrades und der Tradition keinen Anlass, auf den Oberbegriff »Schulsozialarbeit « zu verzichten, zumal dieser auch im internationalen Diskurs identitätsstiftend ist.

Prof. Dr. Susanne Hartmann-Hanff lehrt an der Fachhochschule Frankfurt am Main am Fachbereich "Soziale Arbeit und Gesundheit «. E-Mail suse@fb4.fh-frankfurt.de
Hinsichtlich der Trägerstrukturen ergibt sich für die Schulsozialarbeit bundesweit ein breitgefächertes Bild. Möglich ist sowohl die Anbindung mit Dienst- und Fachaufsicht an den öffentlich-rechtlichen Jugendhilfeträger, als auch an die Schule oder einen Verein oder einen anderen freien Träger. Gebunden an Finanzierungsstruktur, Aufgabenbereich und Interessenausrichtung stellt sich unabhängig von der jeweiligen Trägerorganisation die grundsätzliche Frage nach professionellen Qualitätsstandards und deren Kontrolle.

Eine nicht zu vernachlässigende Annäherungsschwierigkeit an dieses Berufsfeld ist die sich analog zu den Begrifflichkeiten geradezu unübersichtlich ausweitende Vielfalt der Publikationen.

\section{Aufgabenspektrum}

Grundsätzlich setzt Schulsozialarbeit an am zentralen Widerspruch des schulischen Auftrages, nämlich dem demokratischen Gebot gleicher Bildungschancen für alle einerseits und der von der Schule erwarteten Selektion durch Leistungsauslese andererseits.

Vorrangiges Anliegen ist Prävention zur Verhinderung gebrochener Schulkarrieren und damit einhergehender Erschütterungen und Benachteiligungen von individuellen Lebensplänen. Arbeitsschwerpunkte sind die Übergangsbereiche Familie und Schule, Schule und Ausbildung oder Beschäftigung sowie die Optimierung von Sozialisationsprozessen im Schulalltag unter Berücksichtigung geschlechtsspezifischer, soziokultureller und interkultureller Aspekte. Dabei ist die Zielorientierung gleichermaßen individuell, institutionell und gesamtgesellschaftlich.

Verbindliche Arbeitsprinzipien sind die Freiwilligkeit der Teilnahme vonseiten der Schülerinnen und Schüler, die sozialräumliche Vernetzung der Angebote so- wie ein Schweigerecht für die Mitarbeiterinnen und Mitarbeiter. Darüber hinaus sind Mindeststandards im Bezug auf die Personal- und Sachausstattung sowie die Dokumentationspflicht, Fortbildung und Supervision zu gewährleisten, ohne die kein Qualitätsmanagement möglich ist.

Bezugsebenen für die Angebote der Schulsozialarbeit sind: der Unterricht, etwa in Form eines sozialen Beratungssystems für Schüler und Lehrer, Unterstützung durch Teamteaching und Konflikttraining; der nebenunterrichtliche Bereich, etwa durch Projektarbeit mit Peer-group und Erlebnisorientierung, Unterstützung von Klassenfahrten und Schulfesten und der außerunterrichtliche Bereich durch Familien- und stadtteilorientierte Soziale Arbeit.

Zur Förderung von Empowerment und Kompetenzerwerb aufseiten der Schülerinnen und Schüler sind die Handlungsebenen von Schulsoziarbeit sowohl biografieorientiert, also Problembereiche wie psychosexuelle Krisen, Geschlechtsrollenfindung, Nationalität werden fokussiert; schulorientiert, also Beziehungen zu Mitschülern und Lehrern werden thematisiert; als auch berufsorientiert, im Sinne einer Hilfestellung beim Übergang zu Ausbildung oder Beschäftigung und freizeitorientierte Handlungskonzepte der Jugendgruppenarbeit, beispielsweise mit erlebnispädagogischen Inhalten.

Die konkreten Arbeitsstrukturen sollen gemeinsam mit den Lehrern, je nach Schulform und Notwendigkeiten vor Ort, festgelegt werden, damit eine vernetzte Stadtteilarbeit möglich wird.

\section{Ausblick}

Vor dem Hintergrund der unzureichenden gesetzlichen Vorgaben wird plädiert für eine Präzisierung des $\mathbb{1 3}$ a SGB VIII (schulbezogene sozialpädagogische Hilfen und Angebote) und die rechtliche Verankerung einer Kooperationsverpflich- 
tung in den Schulgesetzen. Die inhaltliche Debatte um die Fachsozialarbeit als notwendige professionelle Spezialisierung an Fachhochschulen fordert eine MasterAusbildung in Schulsozialarbeit, unterstützt beispielsweise von der Deutschen Gesellschaft für Sozialarbeit und dem Kooperationsverbund Schulsozialarbeit. Zur Absicherung dieses Berufsfeldes für Sozialarbeiterinnen und Sozialarbeiter sowie Sozialpädagoginnen und Sozialpädagogen fordert der Deutsche Berufsverband für Soziale Arbeit e. V. (DBSH) ein Berufsgesetz.

Erforderlich für ein Gelingen von Schulsozialarbeit ist darüber hinaus eine emanzipatorische Kooperationshaltung von Lehrenden. Hierzu fehlt leider die empirische Basis (vgl.Hanff 2005). Nur wenn Lehrerinnen und Lehrer sich berufspolitisch aktiv an einer Neubestimmung ihres Handlungsauftrages beteiligen, und zwar unter Berücksichtigung der Ressourcen der Jugendhilfe, besteht die Chance, ein Modell von Schule zu entwerfen, deren Pädagogenteam selbstverständlich auch Sozialarbeiterinnen und Sozialarbeiter angehören.

\section{Literatur}

Hartnuß, B./Maykus, St.: Handbuch Kooperation von Jugendhilfe und Schule, Deutscher Verein 2004.

Hanff, S.: Verteilen statt Verzweifeln! Aspekte zur Umkehr der Debatte in: Bassarak, H./Eibeck, B.: (Hg.): Niemanden zurücklassen! Integration durch Schulsozialarbeit in Ganztagsschulen, GEW 2006.

Vernetzungsseite zur Schulsozialarbeit in Deutschland: http://www.schulsozialarbeit.net

\section{Wertvolle Orientierungshilfe}

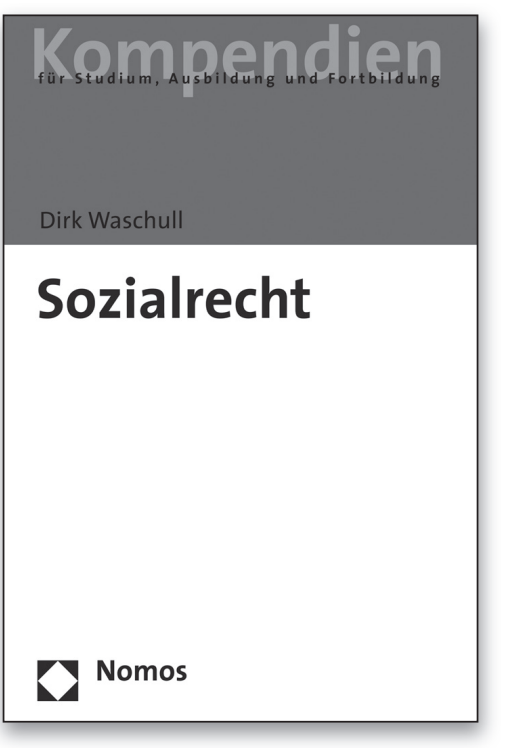

\author{
Sozialrecht \\ Von Prof. Dr. Dirk Waschull, \\ Fachhochschule Münster \\ 2007, ca. 250 S., brosch., ca. 19,- $€$, \\ ISBN 978-3-8329-1824-8 \\ Erscheint Mai 2007
}

Das Sozialrecht ist aufgrund der zahlreichen Reformen kaum noch zu durchschauen. Das neue Kompendium bietet eine methodische und didaktische Einführung in die wesentlichen Themenfelder. Zahlreiche praktische Beispiele und Übersichten ermöglichen den schnellen und einprägsamen Überblick. Dabei stehen aktuelle Fälle zu den Hartz-IV-Gesetzen (SGB II und SGB XII) im Vordergrund.

Die Schwerpunkte der Darstellung:

- Sozialverwaltungsverfahren, insbesondere Stellung der Beteiligten im Verfahren sowie Rücknahme und Widerruf von Verwaltungsakten,

- Leistungserbringerrecht und

[ Übersicht über den sozialgerichtlichen Rechtschutz.

Für den Praktiker in Verwaltungen, bei den freien Trägern und in Selbsthilfeorganisationen bietet das Buch eine wertvolle Orientierungshilfe für die ersten Berufsjahre.

Bitte bestellen Sie bei Ihrer Buchhandlung oder bei: Nomos Verlagsgesellschaft

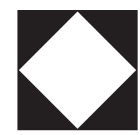

Nomos 